\title{
Modularer Messsystembaukasten für FPGA-basierte Signalverarbeitung
}

\author{
Dominik Laumann, Manuel Selke, Christian Nienhaus, Joachim Doerr \\ CANWAY TECHNOLOGY GMBH, Graf-Zeppelin-Ring 13, D-48346 Ostbevern
}

\begin{abstract}
Zusammenfassung
Das vorliegende Dokument betrachtet die aus der Digitalisierung, der Industrie 4.0 und hochgradig vernetzten Systemen resultierenden Anforderungen an aktuelle und zukünftige Sensor- und Messsysteme für industrielle Produktionsprozesse und zeigt einen Lösungsansatz unter Verwendung eines modularen Baukastensystems basierend auf FPGA Technologie. Systemanforderungen, wie hohe Verfügbarkeit, Zuverlässigkeit sowie Flexibilität und eine intensive Vernetzung von Sensoren und Systemen werden mit verschiedenen Lösungsansätzen von Zustandsüberwachung über Self-X Funktionen bis zu "Soft-Sensoren" adressiert. Diese Lösungsansätze erfordern eine leistungsfähige Signalverarbeitung und hohe Flexibilität auf der Ebene der Sensorschnittstelle, welche optimal durch den Einsatz von FPGAs als Technologie umgesetzt werden kann. Im Folgenden werden die Anforderungen und die Zieltechnologie detaillierter erläutert. Im Anschluss wird das Systemkonzept des Modulbaukastens und die konkrete Umsetzung verschiedener Elektronikmodule vorgestellt. Beispielhafte Anwendungen zeigen die Einsatzmöglichkeiten und Flexibilität des Systems.
\end{abstract}

Die beschriebenen Konzepte sind u. A. im Rahmen des BMBF geförderten Verbundprojekts MoSeS-Pro (FKZ 16ES0422) erarbeitet worden.

Keywords: Modulbaukasten, FPGA, Signalverarbeitung, Sensorschnittstelle

\section{Einführung}

Alle bekannten Bestrebungen für eine zukunftsorientierte Auslegung industrieller Produktionsprozesse, wie z. B. der Industrie 4.0 oder der Digitalisierung, haben gemeinsame Anforderungen auf der Systemebene. Zur Umsetzung dieser "high-level" Anforderungen werden verschiedene Lösungsansätze verfolgt, die entsprechende Anforderungen an Sensorelektronik, Sensorschnittstellen und Messsystemebene bedeuten. Diese Anforderungen werden im Folgenden betrachtet, um im Anschluss ein Lösungskonzept auf Basis von FPGA Technologie vorzustellen.

Eine der Systemanforderungen an industrielle Produktionsprozesse ist die hohe Verfügbarkeit und Zuverlässigkeit. Ein allgemein bekannter Ansatz ist die Zustandsüberwachung von Systemen, die eine Verschleiß- oder Ausfallprädiktion zur Einplanung von Wartungsphasen ermöglicht. Weitere Ansätze sind die frühe Fehlerdetektion und Fehlerkompensation z. B. durch Self-X Eigenschaften von Sensoren, die eine Selbstüberwachung, Selbstkalibrierung oder Selbstheilung ermöglichen [1], und sog. "Soft-Sensoren", die den Ausfall eines Sensors durch intelligente Algorithmen mit Daten noch intakter alternativer Sensoren kompensieren können. Diese Methoden erfordern es, einen möglichst hohen Informationsgehalt aus bestehenden Sensorsignalen zu gewinnen, der häufig über die eigentliche Messaufgabe im Rahmen einer Prozessteuerung hinaus geht. Somit ergeben sich auf Ebene der Sensorschnittstelle zum Teil hohe Anforderungen an die Abtastrate und Signalauflösung, folglich aber auch die Notwendigkeit einer leistungsfähigen Signalverarbeitung und intelligenter Algorithmen zur sensornahen Datenreduktion, um die Kommunikationsinfrastruktur nicht zu überlasten [2].

Eine weitere Systemanforderung ist die Flexibilität von Fertigungs- und Montageprozessen, die eine Adaption von Fertigungssystemen an sich ändernde Aufgaben, sowohl durch Parametrierung des Systems beim Anlagenstillstand als auch durch Rekonfiguration im laufenden Betrieb erfordert. Diese Flexibilität erfordert eine Adaption der Sensor- und Schnittstellenelektronik durch Modularisierung (Flexibilität durch Kombination von Hardwaremodulen) und durch Parametrierung und Rekonfiguration im Feld (Flexibilität durch Kombination von Softwaremodulen). Leistungsreserven dienen dabei zur Vorbereitung 
auf noch nicht bekannte, zukünftige Anwendungsfälle.

Die dritte hier betrachtete Systemanforderung ist die hochgradige Vernetzung, auch unterschiedlicher hierarchischer Ebenen, in industriellen Fertigungsprozessen. Der Ansatz, ein Maximum an Informationsgehalt aus den Prozessen zu gewinnen, erfordert hohe Datenraten und Echtzeitfähigkeit auf verschiedenen Feldbussen und Schnittstellen. Eine zeitliche Synchronisierung der Messstellen ist für eine sensorübergreifende Signalauswertung zwingend erforderlich. Sensorschnittstellen müssen demnach verschiedene aktuelle und zukünftige Kommunikationsprotokolle und Schnittstellen unterstützen, die hohe Datenraten, Echtzeitfähigkeit und eine Zeitsynchronisierung ermöglichen.

Auf Basis dieser Anforderungen an zukünftige Sensorschnittstellen und Messsysteme wurde die Field-Programmable-Gate-Array (FPGA) und System-on-a-Chip (SoC) Technologie als geeignete Zieltechnologie der Elektronik identifiziert. Im Folgenden werden das Konzept und die Umsetzung eines modularen Messsystembaukastens für FPGA-basierte Signalverarbeitung vorgestellt.

\section{FPGA Technologie}

Um die Anforderungen eines vollständig flexiblen Hardwaresystems zu erfüllen, ist die Verwendung von FPGAs derzeit die marktführende Technologie. Dies sind hochkomplexe integrierte digitale Schaltungen zur Adaption an viele verschiedene Anwendungen. FPGAs sind eine Weiterentwicklung von programmierbaren Logikbausteinen. Sie enthalten eine Vielzahl von programmierbaren Logikblöcken (PLB), die komplexe kombinatorische Funktionen ausführen. Diese PLBs sind durch rekonfigurierbare Verbindungen zu einer Gesamtschaltung verbunden. Die Konfiguration wird durch eine Hardwarebeschreibungssprache (HDL) beschrieben. Üblicherweise werden VHDL oder Verilog verwendet. Die HDL beschreibt das Verhalten der Anwendung und wird verwendet, um eine synthetisierbare digitale Schaltung zu erstellen.

Die meisten FPGAs enthalten auch Speicherblöcke zum Zwischenspeichern von Daten. Sie werden oft als SRAM-Speicher implementiert. Ein FPGA Design kann leicht in einen ASIC (Application Specific Integrated Circuit) überführt werden. ASICs sind kundenspezifische Schaltungen, die für eine bestimmte Anwendung ausgelegt sind. Die Produktionskosten sind niedrig, die Einmalkosten jedoch hoch. Die Funktion eines ASICs kann im Betrieb nicht beeinflusst werden. Daher werden FPGAs häufig verwendet, um die Funktionalität vorher zu verifizieren oder sie werden dort eingesetzt, wo hohe Flexibilität erforderlich ist [3].

Moderne Chips kombinieren die FPGA Technologie mit eingebetteten Mikroprozessoren als System-on-a-Chip. Diese Technologie ermöglicht die Kombination leistungsfähiger serieller und paralleler Datenverarbeitung in einem integrierten Schaltkreis [4].

\section{Modulbaukasten Systemkonzept}

Eine Sensorschnittstelle oder generell ein Messsystem lässt sich in vier wesentliche Funktionen unterteilen, die durch ihr Zusammenwirken das Systemverhalten abbilden. Im Sinne des Baukastengedankens wird eine Sensorschnittstelle in vier Subkomponenten mit eben diesen Eigenschaften modularisiert. $A b b .1$ zeigt den schematischen Aufbau einer Sensorschnittstelle und die Auftrennung der vier Teilaufgaben auf die einzelnen Module.

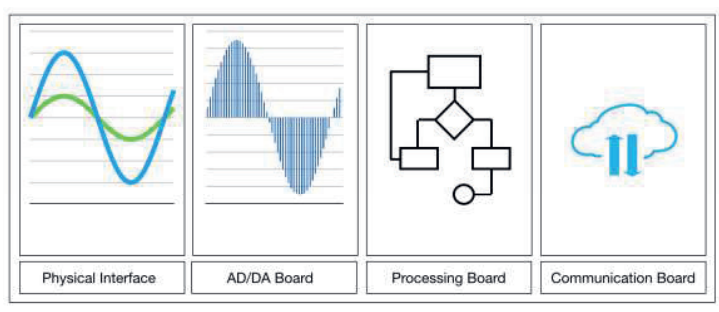

\section{Abb. 1: Schematischer Aufbau einer modula-} ren Sensorschnittstelle

Das Physical Interface dient der Signalkonditionierung des Sensorsignals und stellt notwendige Versorgungsspannungen für die Sensorik zur Verfügung. Der Übergang zwischen analoger und digitaler Welt wird durch ein AD/DA Board realisiert. Die Signalverarbeitung erfolgt auf einem Processing Board. Ein Communication Board dient als Schnittstelle zu einer übergeordneten Steuerung oder Auswertungseinheit. Die Modularisierung auf diese vier Teilaufgaben ermöglicht eine anwendungsspezifische Adaption des Systems durch Kombination der Module. Die Komplexität einzelner Module ist dabei wesentlich reduziert im Vergleich zum Gesamtsystem und generische Module ermöglichen einen hohen Grad der Wiederverwendung.

Im Folgenden werden verschiedene Konzeptbeispiele zum modularen Messsystem erläutert und verschiedene entwickelte Module vorgestellt. Abb. 2 zeigt den Einsatz des Messsystems als Sensorschnittstelle zur sensornahen Signalvorverarbeitung z. B. bei der Zustandsüberwachung. 


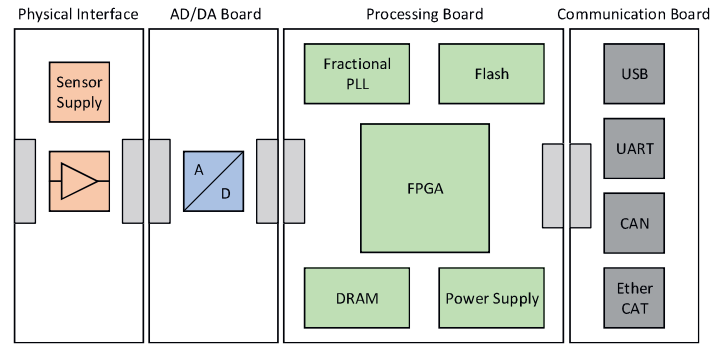

Abb. 2: Sensorschnittstelle für sensornahe Signalvorverarbeitung

Das Physical Interface bildet die analoge Sensorschnittstelle. Hier werden die Signalpegel der Sensoren für eine nachfolgende Verarbeitung angepasst und notwendige Versorgungsspannungen zum Betrieb der Sensorik bereitgestellt. Ein Board mit Analog-Digital-Wandler dient der Digitalisierung des Sensorsignals, das Processing Board stellt einen FPGA für die sensornahe Signalverarbeitung zur Verfügung und das Communication Board ermöglicht die Übertragung von vorverarbeiteten, reduzierten Daten über verschiedene Feldbusschnittstellen. $A b b .3$ zeigt ein erweitertes System mit sensornahem Feedback für Z. B. Self-X Eigenschaften.

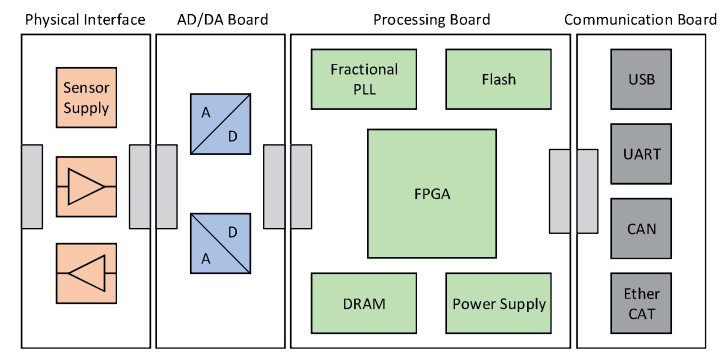

Abb. 3: Sensorschnittstelle mit sensornahem Feedback

Aufgrund der modularen Bauweise müssen zu diesem Zweck nur das Physical Interface und das AD/DA Board ersetzt werden, die Processing und Communication Boards können hardwareseitig identisch verwendet werden und über ein Softwareupdate an die neue Funktion angepasst werden. Das in Abb. 4 dargestellte Konzept erweitert dieses System für TCP/IP basierte Kommunikationsprotokolle und softwarelastige, für die Verarbeitung auf einem Mikroprozessor geeignete Algorithmen.

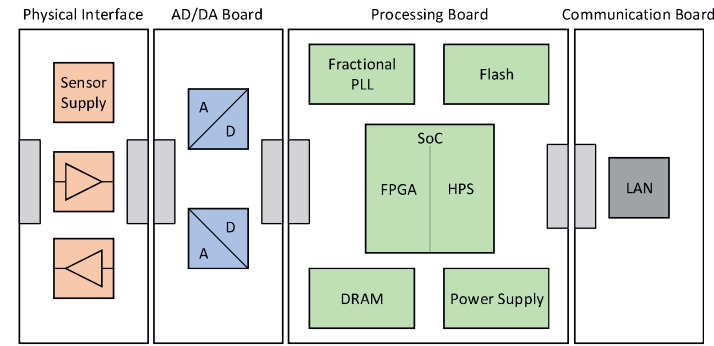

Abb. 4: Sensorschnittstelle mit SoC für TCP/IP-basierte Kommunikation

Dazu wird das FPGA Processing Board durch ein SoC-basiertes Board ersetzt und ein Communication Board mit Ethernet Schnittstelle verwendet. In diesem Fall können das Physical Interface und das AD/DA Board weiterverwendet werden.

\section{Umsetzungsbeispiele der Module}

Im Rahmen des Projekts wurden die Grundlegenden Module, wie FPGA und SoC Processing Boards, sowie verschiedene Interfaceund Kommunikationsmodule umgesetzt und in ausgewählten Anwendungsfällen erprobt. Abb. 5 zeigt ein Processing Board mit einem FPGA der Cyclone V Familie der Firma Intel [5]. Diese FPGA Familie befindet sich im unteren bis mittleren Preis- und Leistungssegment von FPGAs und erfüllt sehr gut die Anforderungen an verfügbare Ressourcen und Performance für die sensornahe Signalverarbeitung.

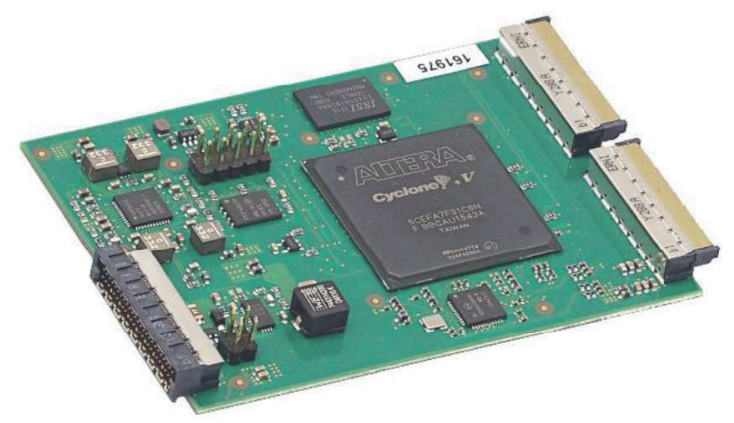

\section{Abb. 5: FPGA Board mit Cyclone V}

Das Processing Board bringt neben dem FPGA auch die gesamte für den Betrieb des FPGAs notwendige Peripherie mit sich, wie Arbeitsspeicher zur Speicherung von Daten und Zwischenergebnissen, einen FlashSpeicher für die FPGA Konfiguration und anwendungsspezifische Parameter und eine konfigurierbare Taktquelle für eine möglichst jitterfreie Abtastung von Sensorsignalen. Neben diesem Processing Board wurde ein weiteres Modul auf SoC-Basis entwickelt, das schnittstellenkompatibel zu dem vorgestellten FPGA-basierten Modul ist. Als SoC wurde hier 
ebenfalls die Cyclone V Familie der Firma Intel gewählt. Die Peripherie ist sehr ähnlich zu der des FPGA Boards, ergänzt um eine Micro SD Karte bzw. einen eMMC Speicher, in denen typischerweise das Dateisystem eines Betriebssystems für den Mikroprozessor Teil des SoCs gespeichert wird.

Für eine exemplarische Anwendung, der Zustandsüberwachung für Verschleißprädiktion an einer Spindelachse, wurde ein AD Board mit zwei verschiedenen Kanaltypen entwickelt.

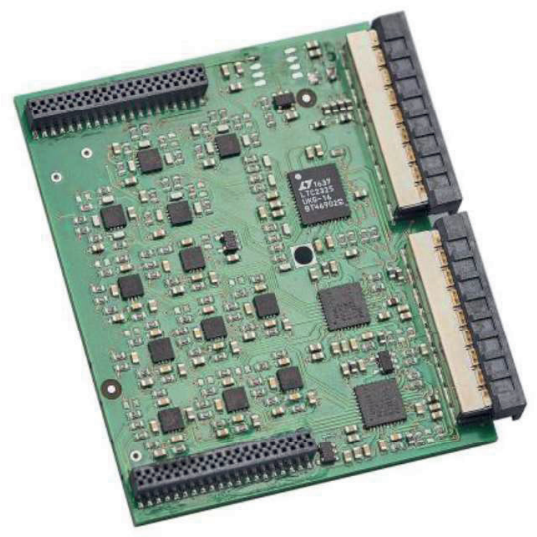

\section{Abb. 6: $\quad A D$ Board}

Das Board stellt insgesamt vier analoge Eingänge mit einer Abtastrate von $5 \mathrm{MS} / \mathrm{s}$ und einer Auflösung von 16 Bit zur Verfügung, die z. B. zur Überwachung von synchron abgetasteten Motorströmen oder Messung von Luftschall eingesetzt werden. Darüber hinaus stehen insgesamt acht analoge Eingänge mit einer Abtastrate von $192 \mathrm{kS} / \mathrm{s}$ und einer Auflösung von 24 Bit ebenfalls mit einer synchronen Abtastung zur Verfügung, mit denen typischerweise Encodersignale und Beschleunigungssensoren gemessen werden können. Ein passendes Physical Interface Board bildet die notwendige Signalkonditionierung und Spannungsversorgung für die Sensoren.

Ein zugehöriges Communication Board ermöglicht die Übertragung von vorverarbeiteten Merkmalen für eine Visualisierung, weitere Verarbeitung oder Speicherung über Ethernet. Auch die Konfiguration des Systems kann so über TCP/IP-basierte Protokolle erfolgen.

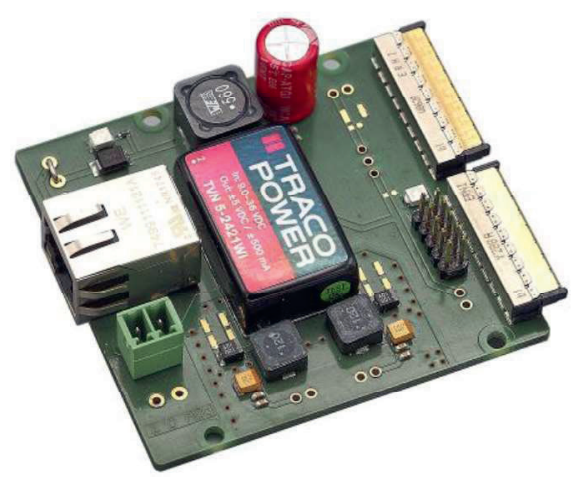

\section{Abb. 7: Communication Board mit Ethernet Schnittstelle}

Um auch komplexere Messsysteme realisieren zu können, lassen sich mehrere FPGA Processing Boards über eine Backplane mit einem SoC Processing Board koppeln. Die FPGA Boards dienen dabei der leistungsfähigen Signalvorverarbeitung, das SoC Board zur übergeordneten Konfiguration, Koordination und Kommunikation. Dazu sind die Boards über die Backplane mittels schneller LVDS Links verbunden. $A b b$. 8 zeigt die Kombination eines FPGA und eines SoC Processing Boards in dem zu diesem Zweck entwickelten Tischgehäuse, das auch die Spannungsversorgung und eine Ethernet Schnittstelle breitstellt.

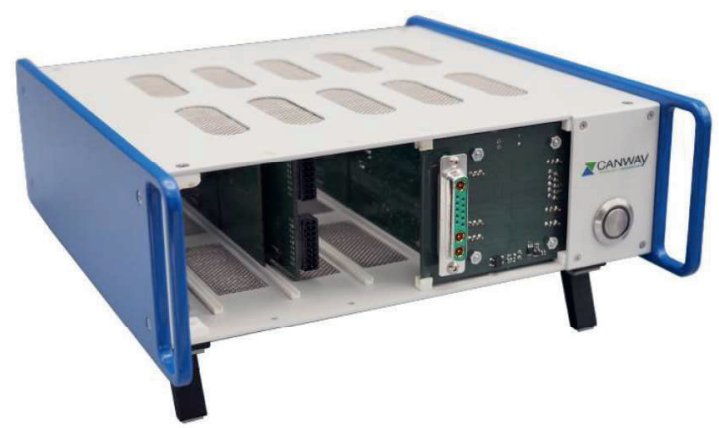

Abb. 8: Komplexes Messsystem im Tischgehäuse

\section{Anwendungsbeispiele}

Im Rahmen des Projekts MoSeS-Pro wurde mit den vorgestellten Elektronikmodulen eine Sensorschnittstelle für die Zustandsüberwachung an einer Spindelachse entwickelt. Abb. 9 zeigt das Gesamtsystem bestehend aus Physical Interface, AD Board, FPGAbasiertem Processing Board und Communication Board. 


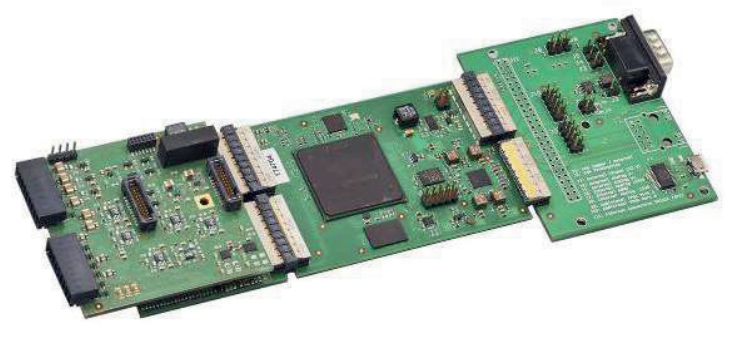

Abb. 9: Sensorschnittstelle für Zustandsüberwachung an Spindelachse

Dieses System bietet die Möglichkeit der Überwachung von Signalen, wie Motorströme, Encoder, Vibration oder Luftschall. Diese Signale können mit Hilfe von Methoden der Signalverarbeitung zur Zustandsüberwachung für eine Verschleißprädiktion genutzt werden. Da diese Algorithmen teilweise hohe Ansprüche an die Signalvorverarbeitung stellen, ist ein FPGA hier optimal geeignet [6].

Die Flexibilität des Systems und die Leistungsfähigkeit der FPGA-basierten Signalverarbeitung kann eindrucksvoll an einem weiteren Beispiel, der Umsetzung eines Netzwerkanalysators, demonstriert werden. Hierzu wurde ein System zur vektoriellen Messung von Übertragungsfunktionen an elektrischen Vierpolen mittels fourier-basierter Technik realisiert. Zu diesem Zweck kann ein zu untersuchendes System mittels verschiedener Signale breitbandig angeregt werden und sowohl der Stimulus als auch die Systemantwort zurückgemessen werden. Die Übertragungsfunktion wird durch Diskrete Fourier Transformation (DFT) im Frequenzbereich ermittelt. Abb. 10 zeigt den schematischen Aufbau des Netzwerkanalysators.

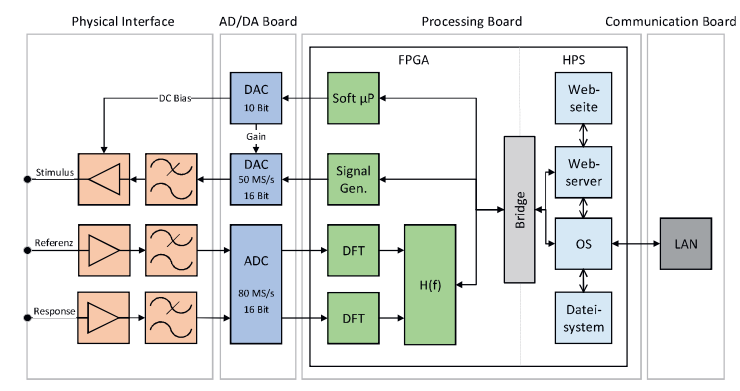

\section{Abb. 9: Netzwerkanalysator (Schematischer Aufbau)}

Die Signalverarbeitung zur Anregung und Auswertung wird im FPGA-Teil eines SoCs durchgeführt. Ein Signalgenerator ermöglicht die Erzeugung verschiedener Anregungssignale (z. B. Pseudozufallsfolgen, Frequenzsweeps oder Multisinus). Das digital generierte Signal wird über einen DA-Wandler mit einer Abtastrate von $50 \mathrm{MS} / \mathrm{s}$ und einer Auflösung von
16 Bit an eine Filter- und Verstärkerstufe ausgegeben und bildet somit den Stimulus der Messung. Ein 2-kanal AD-Wandler mit einer Abtastrate von bis zu $80 \mathrm{MS} / \mathrm{s}$ und einer Auflösung von 16 Bit tastet das Stimulussignal als Referenz und die Systemantwort ab und stellt es dem FPGA zur Verarbeitung zur Verfügung. Ein im FPGA implementierter sog. Softprozessor steuert dabei die Abläufe bei einer Messung und dient der Einstellung von Bias und Gain des Stimulussignals. Die Konfiguration des Systems und die Visualisierung von Messdaten erfolgt über den Mikroprozessorteil (HPS) des SoCs. Auf dem Prozessor kommt ein Embedded Linux Betriebssystem zum Einsatz, welches die Verwendung eines Dateisystems, eines Webservers und die TCP/IP basierte Kommunikation über Ethernet ermöglicht. Eine Webseite dient als Benutzerschnittstelle und ermöglicht die Konfiguration des Systems sowie die Messdatenvisualisierung über einen herkömmlichen Internetbrowser.

\section{Fazit und Ausblick}

Im Rahmen der Untersuchungen wurde ein Konzept für ein modulares Messsystem zur leistungsfähigen, sensornahen Signalvorverarbeitung erstellt und die Funktion an verschiedenen Modulen und Anwendungsfällen demonstriert. Die Modularisierung ermöglicht eine einfache Adaption an verschiedene Anwendungsfälle. Die FPGA Technologie dient hierbei als leistungsstarke, flexible Plattform für sensornahe Signalverarbeitung und ermöglicht eine Rekonfiguration im Feld. Zukünftige Anforderungen an Algorithmik oder Schnittstellen können aufgrund von vorhandenen Leistungsreserven ergänzt werden. Die SoC Technologie bietet darüber hinaus die Ausführung von komplexen, softwarelastigen Algorithmen, die Vorteile eines Betriebssystems und die Kommunikation über TCP/IP-basierte Protokolle.

Im Rahmen dieser Arbeit standen das Systemkonzept und die Entwicklung der Elektronikmodule im Vordergrund. Themen der Softwareinfrastruktur und Konfiguration des Systems, Miniaturisierung und Energieeffizienz müssen Bestandteil weiterer Untersuchungen sein. Dabei soll der bereits in Hardware umgesetzte Baukastengedanke konsequent auf der Ebene der Firmware- und Softwareinfrastruktur weiter verfolgt werden, um die Modularität und Leistungsfähigkeit des Baukastens einem breiteren Anwenderkreis, ohne Erfahrung in der Programmierung von FPGAs, zu eröffnen. Im Rahmen eines "Downsizing" sollen weitere, anwendungsspezifische Module mit reduzierter Größe, geringeren Kosten und minimalem Energiebedarf umgesetzt werden. 


\section{Literaturnachweis}

[1] Andreas Schütze, Nikolai Helwig, Tizian Schneider, Sensors 4.0 - smart sensors and measurement technology enable Industry 4.0 (2018), doi: 10.5194/jsss-7-359-2018

[2] Modulare Sensorsysteme für EchtzeitProzesssteuerung und smarte Zustandsbewertung (MoSeS-Pro), https://www.moses-pro.de/wpcontent/uploads/2016/05/Projektsteckbrief_MoS eS-Pro.pdf, Zugriff: 25.04.2019

[3] M. Selke, C. Nienhaus, D. Laumann, J. Doerr, CANWAY TECHNOLOGY GMBH, Ostbevern (Germany), Universal Sensor Interface for High Performance Signal Processing Based on FPGA Technology (2017), doi: 10.5162/sensor2017/B2.2

[4] Prof. Dr.-Ing. Frank Kesel, Dr. Ruben Bartholomä, Entwurf von digitalen Schaltungen und Systemen mit HDLs und FPGAs, Oldenbourg Verlag München, 3. Auflage 2013, ISBN 978-3-486-73181-1

[5] Intel/Altera Inc., San Jose 2018, Cyclone V Device Handbook

[6] U. Meyer-Baese, Digital Signal processing with Field Programmable Gate Arrays, Springer Verlag, 4. Auflage 2014, ISBN 978-3-64245308-3 